\title{
SCATTERING OF WATER WAVES BY A VERTICAL WALL WITH GAPS
}

\author{
SUDESHNA BANERJEA ${ }^{1}$
}

(Received 15 October 1993; revised 23 August 1994)

\begin{abstract}
This paper is concerned with a reinvestigation of the problem of water wave scattering by a wall with multiple gaps by using the solution of a singular integral equation with a combination of logarithmic and power (Cauchy-type) kernels in disjoint multiple intervals. Use of Havelock's expansion of water wave potential reduces the problem to such an integral equation in the horizontal velocity across the gaps. The solution of the integral equation is obtained by utilizing the solutions of Cauchy-type integral equations in $(0, \infty)$ and also in multiple disjoint intervals. An explicit expression for the reflection coefficient is obtained for a wall with $n$ gaps and supplemented by numerical results for up to three gaps.
\end{abstract}

\section{Introduction}

The study of the two-dimensional problem of scattering of surface waves by a thin vertical barrier was initiated more than forty years back and a number of mathematical techniques have been utilized to solve it for various configurations of the barrier. The problem for a completely submerged vertical barrier extending infinitely downwards was considered by Dean [2], the partially immersed vertical barrier by Ursell [12] and the completely submerged plate problem by Evans [3]. The more general problem of scattering of water waves by a thin vertical wall with an arbitrary number of gaps was considered by Lewin [5], Mei [7], Porter [10, 9] and Macaskill [6]. Essentially they used a reduction technique or an integral equation formulation based on a suitable application of Green's integral theorem in the fluid region to solve these problems.

There is an alternative integral equation formulation based on Havelock's [4] expansion of water wave potential. In this method the problem is reduced to a

\footnotetext{
${ }^{1}$ Department of Mathematics, Jadavpur University, Calcutta - 700 032, India.

(C) Australian Mathematical Society, 1996, Serial-fee code 0334-2700/96
} 
singular integral equation having the general form

$$
\int_{L} f(t)\left[K \ln \left|\frac{x-t}{x+t}\right|+\frac{1}{x+t}+\frac{1}{x-t}\right] d t=g(x), \quad x \in L
$$

where $g(x)$ is a prescribed function and $L$ may consist of a single or multiple intervals. The function $f(x)$ is the unknown horizontal component of velocity across the gaps and as such it has integrable singularities at the end points of $L$ except at 0 and $\infty$ if these belong to $L$. For the scattering problem involving a partially immersed or completely submerged thin vertical plate, $L$ is either $(0, a)$ or $(b, \infty)$. In these cases (1.1) can be reduced to another singular integral equation with Cauchy-type kernel whose solution is well-known (see Ursell [12]). However, for the scattering problem involving a thin vertical wall with a single gap or multiple gaps, $L$ consists of a single or multiple intervals. For these cases it is not possible to reduce (1.1) to an integral equation with Cauchy kernel, and as such the solution of (1.1) needs special consideration. This motivated us to study the integral equation (1.1) (with $g(x)=0$ ) when $L$ consists of disjoint multiple intervals, and thereby to reinvestigate the problem of water wave scattering by a vertical wall, with multiple gaps. The method of solution of the integral equation presented here seems to be very general. The solution of the integral equation involves $n$ arbitrary constants which are determined in the course of solution of the physical problem.

The multiple-gap problem considered by Porter [10] was reduced to an integral equation of the first kind in multiple intervals by using a reduction technique. The kernel of the integral equation has only the Cauchy-type singularity. The function satisfying this integral equation has the property that it is bounded at the end points of each interval. The solution of this integral equation was then obtained by using the function-theoretic method. In the process of solving this integral equation, no extra arbitrary constants were generated. However for $n$ gaps, $2 n$ arbitrary constants appeared in the right side of the above mentioned integral equation in a natural manner so that its solution also involves $2 n$ arbitrary constants which were determined from certain solvability criteria together with a set of requirements at the barrier edges (see Porter [10]).

The single-gap problem was earlier treated by Porter [9] using the reduction technique as well as the integral equation formulation based on Green's integral theorem. Tuck [11] used a matched asymptotic method to investigate the single-gap problem when the gap is narrow in the sense that the gap length is very small compared to the depth of submergence of the midpoint of the gap below the mean free surface.

The integral equation (1.1) with $L$ consisting of $(0, a)$ and $(b, \infty)$ as well as $(a, b)$ was obtained recently by Banerjea and Mandal [1] in a straightforward manner by using the solution of the Cauchy-type integral equation in $(0, \infty)$. In the present paper, the solution of (1.1) with $g(x)=0$ is obtained when $L$ consists of the union of 
$n$ disjoint finite intervals $\left(a_{i}, b_{i}\right), i=1,2, \ldots, n$ by a simple straightforward method based on utilizing the solutions of Cauchy type integral equations in $(0, \infty)$ and in the union of $(n+1)$ disjoint intervals $\left(0, a_{1}\right),\left(b_{i-1}, a_{i}\right) i=2, \ldots, n$ and $\left(b_{n}, \infty\right)$. This Cauchy type integral equation is then solved by function-theoretic method. The solution of the homogeneous form of (1.1) is then used to reinvestigate the aforesaid two-dimensional $n$ gap problem. An explicit expression for the reflection coefficient is also obtained, and the numerical results are presented graphically for a wall with a single gap, two gaps and three gaps.

In the course of evaluation of the reflection coefficient numerically, Gauss quadrature formula is used appropriately to calculate the various singular integrals appearing in the results, and also a system of $n$ linear equations with complex coefficients has been solved by standard methods. It may be noted that while Lewin [5], Mei [7] and Porter [10] did not present any numerical results, Macaskill [6] presented the numerical results for the transmission coefficient graphically for a single gap as well as for two gaps in a vertical wall in deep water. He used an integral equation formulation based on an application of Green's integral theorem, and utilized the collocation method for numerical solution of the integral equation, which thus involved inversion of a matrix of quite large order. The present method appears to be straightforward. The numerical computations can be carried out in a routine manner for any number of gaps and involves inversion of a matrix of order only $n$, the number of gaps.

For $n>1$, the gap sizes are first taken to be equal while the lengths of the portions of the wall between the gaps are also taken to be equal. For $n=1$ it is found that the results for a single gap agrees with Porter [9]. Also it is observed that for a wall with two or three gaps, the reflection coefficient is very small for certain values of the wave number. This implies that at certain wave numbers, almost total transmission of wave energy occurs. The reflection coefficient for a wall with two gaps has also been computed numerically for two cases, namely: i) when the gap lengths are equal but the lengths of the portions of the wall between the gaps are unequal; ii) when the gap lengths are unequal but the lengths of the portions of the wall between the gaps are equal. In the first case the numerical results for the reflection coefficient were found to be in agreement with that of Macaskill [6]. In the second case it has been observed that when the gap sizes are large and the ratio between the lengths of lower gap and the upper gap increases, then for certain wave numbers, perfect transmission occurs.

\section{Statement and formulation of the problem}

We consider a thin vertical wall $x=0,0<y<\infty$ with $n$ gaps $G_{i}$, given by $x=0, a_{i}<y<b_{i}, i=1,2, \ldots, n$ in deep water occupying the region $y \geq 0$ with $y=0$ as the mean free surface. A train of surface waves of frequency $\sigma$ is incident 
upon the wall from negative infinity, it is then partially transmitted through the gaps and partially reflected by the wall. Under the usual assumption of linear theory, the time harmonic motion in the fluid region can be described by a velocity potential $\operatorname{Re}\{\phi(x, y) \exp (-i \sigma t)\}$, where $\phi$ satisfies

$$
\nabla^{2} \phi=0 \text { in the fluid region; }
$$

the linearised free surface condition

$$
K \phi+\phi_{y}=0 \text { on } y=0,
$$

$K=\sigma^{2} / g, g$ representing gravity; the condition on the wall,

$$
\phi_{x}=0 \quad \text { on } x=0, \quad y \in B \equiv \cup_{i=1}^{n+1} B_{i},
$$

where $B_{1}=\left(0, a_{1}\right), B_{j}=\left(b_{j-1}, a_{j}\right), j=2, \ldots, n$ and $B_{n+1}=\left(b_{n}, \infty\right)$; the edge condition

$$
r^{1 / 2} \nabla \phi \quad \text { is bounded as } \quad r \rightarrow 0,
$$

where $r$ is the distance from the points $\left(0, a_{i}\right),\left(0, b_{i}\right), i=1,2, \ldots, n$; the bottom condition

$$
\nabla \phi \rightarrow 0 \quad \text { as } \quad y \rightarrow \infty
$$

and the infinity requirement

$$
\phi \sim \begin{cases}\exp (-K y+i K x)+R \exp (-K y-i K x) & \text { as } \quad x \rightarrow-\infty \\ T \exp (-K y+i K x) & \text { as } \quad x \rightarrow \infty\end{cases}
$$

where $R$ and $T$ are the reflection and transmission coefficients respectively. Our aim is to evaluate $R$ and $T$.

\section{Reduction of the problem to a singular integral equation}

Let

$$
\frac{\partial \phi}{\partial x}( \pm 0, y)=F(y), \quad 0<y<\infty .
$$

Then

$$
F(y)= \begin{cases}0, & y \in B \\ f(y), & y \in G,\end{cases}
$$

where $f(y)$ is an unknown function of $y$ and $G \equiv \cup_{i=1}^{n} G_{i}$. Moreover, because of (2.4),

$$
f(y)=\left\{\begin{array}{lll}
\mathrm{O}\left(\left|y-a_{i}\right|^{-1 / 2}\right) & \text { as } & y \rightarrow a_{i} \\
\mathrm{O}\left(\left|b_{i}-y\right|^{-1 / 2}\right) & \text { as } & y \rightarrow b_{i}, i=1,2, \ldots, n .
\end{array}\right.
$$


It may be noted that $G \cup B=(0, \infty)$.

By Havelock's [4] expansion of the water wave potential, a suitable representation for $\phi(x, y)$ satisfying (2.1), (2.2), (2.5) and (2.6) is given by

$$
\phi(x, y)= \begin{cases}T \exp (-K y+i K x)+\int_{0}^{\infty} A(k) M(k, y) \exp (-k y) d k, & x>0, \\ \exp (-K y+i K x)+R \exp (-K y-i K x)+ & x<0 \\ \int_{0}^{\infty} B(k) M(k, y) \exp (k y) d k, & \end{cases}
$$

where

$$
M(k, y)=k \cos k y-K \sin k y .
$$

Utilizing (3.4) in (3.1) and Havelock's [4] theorem, we find that

$$
T=1-R=-2 i \int_{0}^{\infty} F(y) \exp (-K y) d y
$$

and

$$
-k A(k)=k B(k)=\frac{2}{\pi} \frac{1}{k^{2}+K^{2}} \int_{0}^{\infty} F(y) M(k, y) d y .
$$

An integral equation for $f(y)$ is now obtained from the fact that $\phi(x, y)$ is continuous across the gaps, so that $\phi\left({ }_{+} 0, y\right)=\phi(-0, y), \quad y \in G$. This gives

$$
-\frac{\pi}{2} R \exp (-K y)=\int_{0}^{\infty} \frac{M(k, y)}{k\left(k^{2}+K^{2}\right)}\left[\int_{G} f(u) M(k, u) d u\right] d k, \quad y \in G .
$$

On applying the operator $\frac{d}{d y}+K$ to (3.8), we obtain the singular integral equation for $f(y)$,

$$
\int_{G} f(u)\left[K \ln \left|\frac{y-u}{y+u}\right|+\frac{1}{y+u}+\frac{1}{y-u}\right] d u=0, \quad y \in G,
$$

where the integral is in the sense of Cauchy principal value.

It may be noted that (3.9) is the homogeneous form of (1.1) and $L=G$.

\section{Solution of the integral equation}

In this section we obtain the solution of (3.9). The solution of the nonhomogeneous form of (3.9) can be obtained in a similar manner.

Equation (3.9) can be written as

$$
\int_{0}^{\infty} F(u)\left[K \ln \left|\frac{y-u}{y+u}\right|+\frac{1}{y+u}+\frac{1}{y-u}\right] d u=H(y), \quad 0<y<\infty
$$


where

$$
H(y)= \begin{cases}0, & y \in G \\ h(y), & y \in B\end{cases}
$$

with $h(y)$ unknown at this stage and $F(y)$ given by (3.2).

Due to (3.3) we find that

$$
h(y)=\left\{\begin{array}{lll}
\mathrm{O}\left(\left|y-a_{i}\right|^{-1 / 2}\right) & \text { as } & y \rightarrow a_{i} \\
\mathrm{O}\left(\left|y-b_{i}\right|^{-1 / 2}\right) & \text { as } \quad y \rightarrow b_{i}, i=1,2, \ldots, n .
\end{array}\right.
$$

Writing

$$
\lambda(u)=K \int_{0}^{u} F(x) d x+F(u)
$$

reduces $(4.1)$ to

$$
\int_{0}^{\infty} \frac{\lambda(u)}{y^{2}-u^{2}} d u=\frac{H(y)}{2 y}, \quad 0<y<\infty .
$$

The solution of (4.5) can be obtained (see [11]) as

$$
\lambda(u)=\frac{2}{\pi^{2}} \int_{0}^{\infty} \frac{t H(t)}{t^{2}-u^{2}} d t+s, \quad 0<u<\infty
$$

where $s$ is an arbitrary constant. By (4.2), this reduces to

$$
\lambda(u)=\frac{2}{\pi^{2}} \int_{B} \frac{t h(t)}{t^{2}-u^{2}} d t+s .
$$

The integrals in (4.5), (4.6) and (4.7) are in the sense of Cauchy principal values. The same is the case in some subsequent integrals also. Thus using (4.5) we find for $0<y<\infty$ that

$$
F(y)=\frac{d}{d y}\left[\exp (-K y) \int_{0}^{y} \exp (K u) \lambda(u) d u\right] .
$$

However in (4.8) and (4.7), $\lambda(u)$ involves the unknown function $h(t)$. An integral equation for $h(t)$ can be obtained from the fact that $F(y)$ vanishes for $y \in B$, so that after integration

$$
\int_{0}^{y} \exp (K u) \lambda(u) d u=A_{i} \exp (K y), \quad y \in B_{i}, \quad i=1,2, \ldots, n+1,
$$

where $A_{i}(i=1,2, \ldots, n+1)$ are arbitrary constants. This gives

$$
\int_{B} \frac{2 t h(t)}{t^{2}-y^{2}} d t=c_{i} \quad \text { for } \quad y \in B_{i}, \quad i=1,2, \ldots, n+1,
$$


where

$$
c_{i}=\pi^{2}\left(K A_{i}-s\right) .
$$

Noting that $B_{n+1} \equiv\left(b_{n}, \infty\right)$, we can make $y \rightarrow \infty$ in (4.10) to obtain

$$
c_{n+1}=0 \text {. }
$$

The solution of (4.10) satisfying (4.3) is obtained in the Appendix and is given, after noting (4.12), by

$$
\begin{gathered}
h(u)=\frac{(-1)^{n+1-r}}{R_{r}(u)}\left[\sum_{k=1}^{n} \pi P_{k-1} u^{2 k-1}-\frac{2}{\pi^{2}} \sum_{k=1}^{n} c_{k} u F_{k}(u)\right], \\
u \in B_{r}, \quad r=1,2, \ldots, n+1,
\end{gathered}
$$

where

$$
\begin{gathered}
R_{r}(u)=\prod_{j=1}^{n}\left|\left(u^{2}-a_{j}^{2}\right)\left(u^{2}-b_{j}^{2}\right)\right|^{1 / 2}, \\
F_{k}(u)=(-1)^{n+1-k} \int_{B_{k}} \frac{R_{k}(v)}{v^{2}-u^{2}} d v, \quad k=1,2, \ldots, n
\end{gathered}
$$

and $p_{k}, k=0,1, \ldots, n-1$ are $n$ arbitrary constants. Equations for determining various constants can be obtained by using $F(y)=0$ for $y \in B_{r}, r=1,2, \ldots, n+1$. Thus we obtain from (4.8), (4.7) and (4.2) that

$$
\frac{d}{d y}\left[\exp (-K y)\left\{\int_{0}^{y} \exp (K u) \frac{2}{\pi^{2}}\left(\int_{B} \frac{t h(t)}{t^{2}-u^{2}} d t\right) d u-\frac{s}{K}\right\}\right]=0 .
$$

By (4.10), this gives after simplification for $y \in B_{1}$ that

$$
\left(\frac{c_{1}}{\pi^{2}}+s\right) \frac{d}{d y} \exp (-K y)=0
$$

so that

$$
\frac{c_{1}}{\pi^{2}}+s=0
$$

Again for $y \in B_{2}$, we find from (4.16) after simplification using (4.10) and (4.17), that

$$
\begin{aligned}
& {\left[\frac{c_{1}}{\pi^{2} K} \exp \left(K a_{1}\right)-\frac{c_{2}}{\pi^{2} K} \exp \left(K b_{1}\right)\right.} \\
& \left.\quad+\int_{a_{1}}^{b_{1}} \exp (K u) \frac{2}{\pi^{2}}\left(\int_{B} \frac{t h(t)}{t^{2}-u^{2}} d t\right) d u\right] \frac{d}{d y} \exp (-K y)=0 .
\end{aligned}
$$


This implies that the term in brackets vanishes. The $t$-integral can be further simplified after using (4.13) to (4.15), and this gives

$$
\frac{c_{1}}{\pi^{2} K} \exp \left(K a_{1}\right)-\frac{c_{2}}{\pi^{2} K} \exp \left(K b_{1}\right)+(-1)^{n-1} \int_{a_{1}}^{b_{1}} \frac{u \exp (K u)}{R_{1}(u)} N(u) d u,
$$

where

$$
N(u)=\sum_{k=1}^{n}\left[p_{k-1} u^{2 k-2}-\frac{2}{\pi} \frac{c_{k}}{\pi^{2}} F_{k}(u)\right] .
$$

Applying the same procedure in (4.16) successively for $y \in B_{r}, r=3,4, \ldots, n$, we find that

$$
\begin{aligned}
& {\left[\frac{c_{r}}{\pi^{2} K} \exp \left(K a_{r}\right)-\frac{c_{r+1}}{\pi^{2} K} \exp \left(K b_{r}\right)\right.} \\
& \left.\quad+(-1)^{n-r} \int_{a_{r}}^{b_{r}} \frac{u \exp (K u)}{R_{r}(u)} N(u) d u\right] \frac{d}{d y} \exp (-K y)=0
\end{aligned}
$$

which implies that the term in brackets vanishes. Therefore for $r=1,2, \ldots, n$ we obtain

$$
\frac{c_{r}}{\pi^{2} K} \exp \left(K a_{r}\right)-\frac{c_{r+1}}{\pi^{2} K} \exp \left(K b_{r}\right)+(-1)^{n-r} \int_{a_{r}}^{b_{r}} \frac{u \exp (K u)}{R_{r}(u)} N(u) d u=0 .
$$

Finally the solution of (3.9) is obtained from (4.8) after using (4.7), (4.13) to (4.15), (4.17) and (4.20), as

$$
\begin{gathered}
f(y)=\frac{d}{d y} \exp (-K y)\left[\frac{c_{r}}{\pi^{2} K} \exp \left(K a_{r}\right)+(-1)^{n-r} \int_{a_{r}}^{y} \frac{u \exp (K u)}{R_{r}(u)} N(u) d u\right], \\
a_{r}<y<b_{r}, \quad r=1,2, \ldots, n .
\end{gathered}
$$

The expression in (4.21) involves $2 n$ arbitrary constants. These $2 n$ constants satisfy $n$ linear equations (4.20). So we need another set of $n$ relations so as to determine them completely. These will be obtained in the next section, and the reflection coefficient will be determined in a natural way.

\section{Reflection and transmission coefficients}

Here we shall obtain another set of $n$ equations for the constants $c_{k}, p_{k-1}$ ( $k=$ $1,2, \ldots, n)$. To achieve this we substitute $f(y)$ from (4.21) in the original integral equation (3.8). The various integrals in the right side of (3.8) can be simplified giving 
the following identity for $a_{m}<y<b_{m},(m=1,2, \ldots, n)$,

$$
\begin{aligned}
{[R} & -\sum_{j=1}^{m}(-1)^{n+1-j} \int_{B_{j}} \frac{2 u N(u)}{R_{j}(u)} \sinh (K u) d u \\
& \left.+\sum_{j=m+1}^{n+1}(-1)^{n+1-j} \int_{B_{j}} \frac{u N(u)}{R_{j}(u)} \exp (-K u) d u\right] \exp (-K y)=0 .
\end{aligned}
$$

This gives

$$
\begin{aligned}
R= & -\sum_{j=1}^{m}(-1)^{n-j} \int_{B_{j}} \frac{2 u N(u)}{R_{j}(u)} \sinh (K u) d u \\
& +\sum_{j=m+1}^{n+1}(-1)^{n-j} \int_{B_{j}} \frac{u N(u)}{R_{j}(u)} \exp (-K u) d u .
\end{aligned}
$$

Again using (3.2) and (4.20) in (3.6), we find that

$$
\begin{array}{r}
R=1-i \sum_{j=1}^{n}\left[\frac{c_{j}}{\pi^{2} K} \exp \left(-K a_{j}\right)-\frac{c_{j+1}}{\pi^{2} K} \exp \left(-K b_{j}\right)\right. \\
\left.-\int_{a_{j}}^{b_{j}} \frac{(-1)^{n-j} u N(u)}{R_{j}(u)} \exp (-K u) d u\right] .
\end{array}
$$

Equating the right-hand sides of (5.1) and (5.2), we now obtain another set of $n$ relations for the $2 n$ arbitrary constants $c_{k}, p_{k-1}(k=1,2, \ldots, n)$, the first set of $n$ relations being given by (4.20). Thus $2 n$ unknown constants are known completely. Finally $R$ is obtained either from (5.1) or from (5.2) and $T$ is given by $1-R$.

Special case $n=1 \quad$ This corresponds to the problem of water wave transmission through a gap in a wall. In this case $R$ is given by

$$
R=i I /(J+i I)
$$

where

$$
\begin{aligned}
J & =\frac{\exp \left(-K a_{1}\right)}{K}+\alpha_{2}(K)-\frac{2}{\pi} \alpha_{2}\left(K, F_{1}\right), \\
I & =\alpha_{1}(K)-\alpha_{3}(K)-\frac{2}{\pi} \alpha_{1}\left(K, F_{1}\right)+\frac{2}{\pi} \alpha_{3}\left(K, F_{1}\right), \\
\alpha_{2}(-K) & =\exp \left(K a_{1}\right)+\frac{2}{\pi} \alpha_{2}\left(-K, F_{1}\right), \\
\alpha_{i}\left(K, F_{1}\right) & =\int_{t_{i}} \frac{u F_{1}(u)}{R(u)} \exp (-K u) d u,
\end{aligned}
$$




$$
\begin{aligned}
F_{1}(u) & =\int_{0}^{a_{1}} \frac{R(v)}{v^{2}-u^{2}} d v, \\
\alpha_{i}(K) & \equiv \alpha_{i}(K, 1), \quad R(v)=\left|\left(v^{2}-a_{1}^{2}\right)\left(v^{2}-b_{1}^{2}\right)\right|^{1 / 2}
\end{aligned}
$$

and

$$
t_{i}=\left\{\begin{array}{ll}
\left(-a_{1}, a_{1}\right), & i=1 \\
\left(a_{1}, b_{1}\right), & i=2 . \\
\left(b_{1}, \infty\right), & i=3
\end{array} .\right.
$$

This result agrees with Porter [9] except for a trivial mistake in sign in the expression for $\delta$. It may be noted that making $a_{1} \rightarrow 0$ ( $b_{1}$ fixed) and $b_{1} \rightarrow \infty$ ( $a_{1}$ fixed), the results for the scattering problem involving a thin vertical barrier completely or partially immersed in deep water can be deduced (see Ursell [12]).

\section{Discussion}

The reflection coefficient $|R|$ has been computed numerically for three cases, namely when the wall has a single, two and three gaps. For simplicity we first assume that the gaps are of equal length and except for the portion extending infinitely downwards, all the portions of the wall inside the water region are of the same length. Let $h$ be the depth of submergence of the midpoint of the first gap and $\mu$ be the ratio of the width of the gap to $h$. Then $a_{1}=(2-\mu) h / 2, b_{1}=(2+\mu) h / 2, a_{2}=2 h$, $b_{2}=2 b_{1}, a_{3}=(6+\mu) h / 2, b_{3}=3 b_{1}$. We have computed $|R|$ for these three cases, for $K h=0.1,0.2, \ldots, 4.0$, and $\mu=0.1,0.5,1.0,1.5$. The numerical results are presented graphically for a single gap in Figure 1, two gaps in Figure 2 and three gaps in Figure 3.

It is observed from Figures 1,2 and 3 that $|R|$ decreases at first, reaches a minimum value and then again increases as the wave number $K h$ increases. For large values of the wave number, it is observed that $|R|$ almost becomes unity. This is expected because short waves are confined near the free surface and so they are almost totally reflected by the uppermost portion of the wall, as the wave is practically confined within a thin layer near the surface. Also, as $\mu$ increases from 0.1 to 1.5 , it is found in all three cases that the aforesaid minimum value of $|R|$ decreases. Again, from Figures 1,2 and 3 , it is found that for $\mu=1.5$, the minimum value of $|R|$ is closer to zero for a wall with three gaps than for a wall with one or two gaps. Thus, for a fixed $\mu$, that is, when the gap sizes are fixed, the minimum value of $|R|$ moves closer to zero as the number of gaps in the wall increases. This shows that as the gap size increases and the number of gaps increases, there exists a certain wave number for which almost total transmission of wave energy occurs. Again, for a wall with a single gap, it has been checked that for a small gap $(\mu=0.1)$ there is almost total reflection 


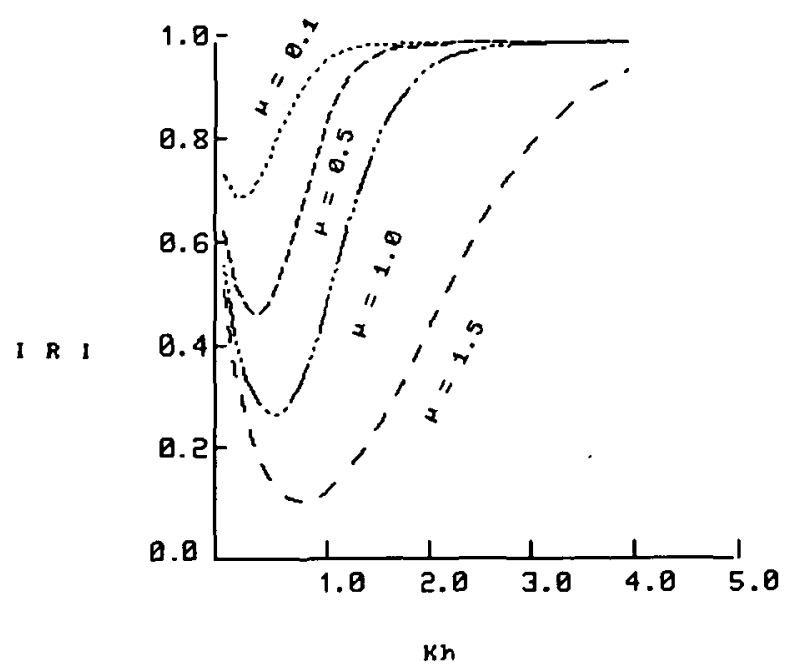

FIGURE $1 .|R|$ vs. $K h$ for a single gap

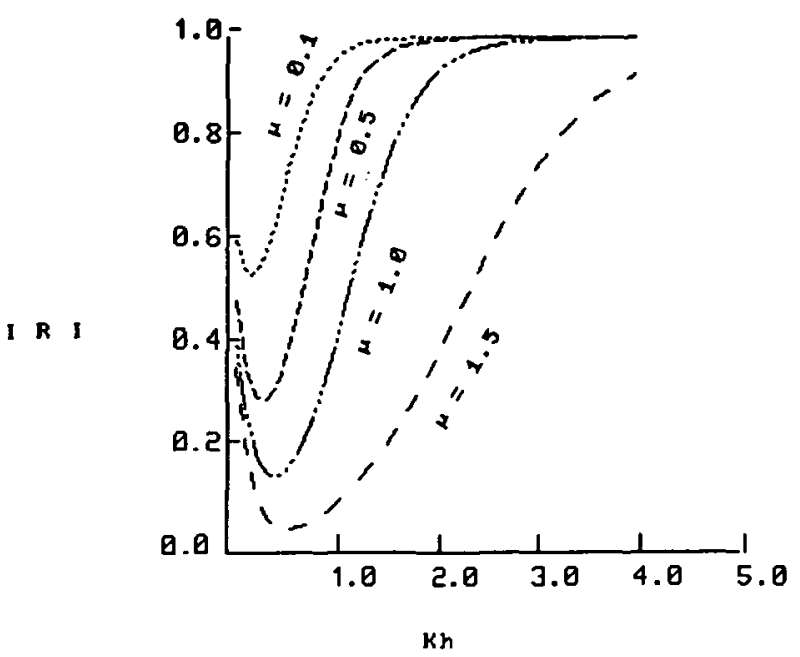

FIGURE 2. $|R|$ vs. $K h$ for two gaps

for small values of $K h$. This observation is in good agreement with the small gap theory of Tuck [11]. This is also somewhat apparent from the curve for $\mu=0.1$ of Figure 1. 


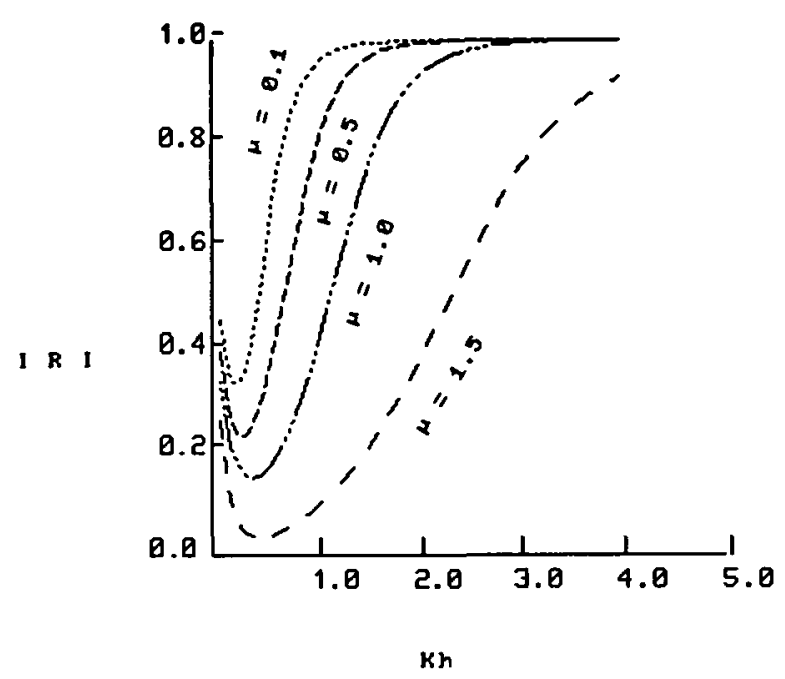

FIGURE 3. $|R|$ vs. $K h$ for three gaps

The reflection coefficient $|R|$ for two gaps has also been computed numerically for the cases

(i) when the gap lengths are equal but the lengths of the portions of the wall are unequal;

(ii) when the gap lengths are unequal and the lengths of the portions of the wall (excepting the one extending infinitely downwards) are equal.

Let $\alpha$ denote the ratio of the distance between the centres of the two gaps to $h$. Then $a_{1}=(2-\mu) h / 2, b_{1}=(2+\mu) h / 2, a_{2}=h(1+\alpha-\mu / 2)$ and $b_{2}=h(1+\alpha+\mu / 2)$ for case (i). For this case $|R|$ is plotted graphically against $K h$ in Figures 4,5 and 5a.

It is observed from Figure 4 that for $\mu=0.1, \alpha=0.2,|R|$ first decreases and then increases to unity asymptotically. It has also been checked that for very small values of $K h,|R|$ is nearly unity. This shows that when the gap lengths are small $(\mu=0.1)$ and the two gaps are very close to each other $(\alpha=0.2)$, then there is almost total reflection for very small values of the wave number. This phenomenon was also observed by Macaskill [6] (Figure 9 there).

For $\mu=1.0, \alpha=12.5$ it is observed from Figure 5 that $|R|$ at first decreases and then increases as $K h$ increases. However, for small values of $K h$, a small indentation which is not very pronounced can be observed in the graph of $|R|$. For $\alpha=50$, that is, when the lower gap is at considerably large distance from the upper gap, $|R|$ is plotted in an enlarged manner for small values of $K h$ (up to 0.6) in Figure 5a. From this figure it is observed that $|R|$ at first decreases, then increases slightly and then 
again decreases for small $K h$. As $K h$ further increases $|R|$ also decreases further

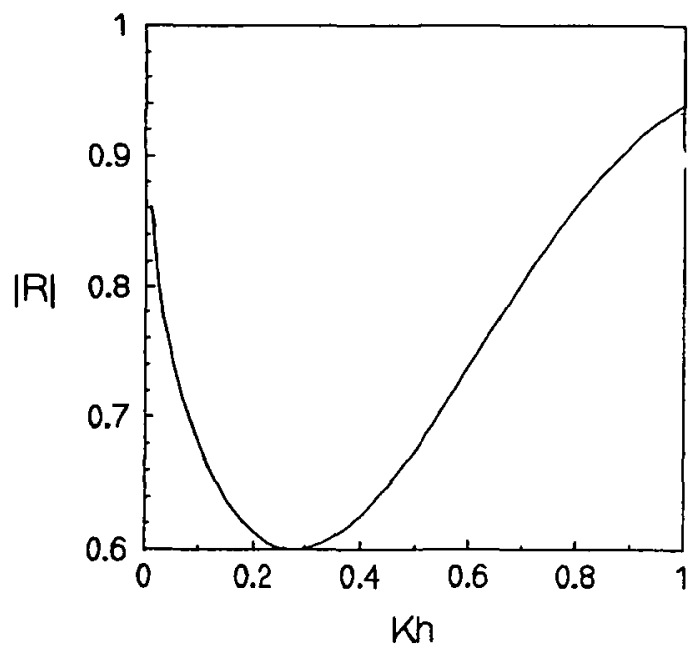

FIGURE 4. Reflection of water waves by a wall with two gaps $\mu=0.1, \alpha=0.2$

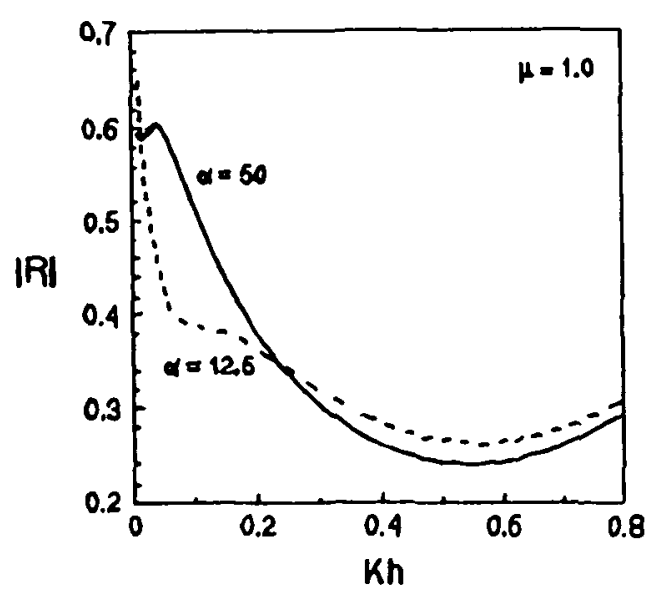

FIGURE 5. Reflection of water waves by a wall with two gaps

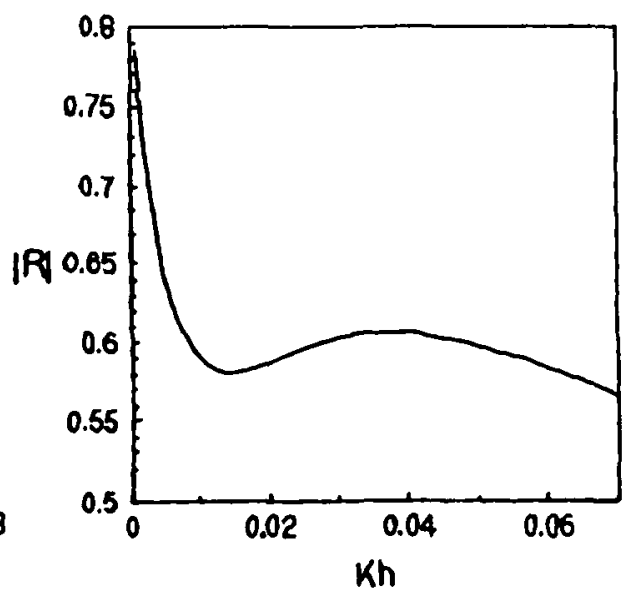

FIGURE 5A. Reflection of water waves by a wall with two gaps $\mu=1.0, \alpha=50$

up to 0.24 (at $K h=0.54$ ) and then increases asymptotically to unity (see Figure 5). Thus for large gaps and large separation distance between the two gaps, a dip appears in the reflection coefficient for small values of $K h$. This phenomenon which may be attributed to some resonance effect taking place owing to the interaction of the flow with the two gaps, was also observed by Macaskill [6] (Figure 8 there). 


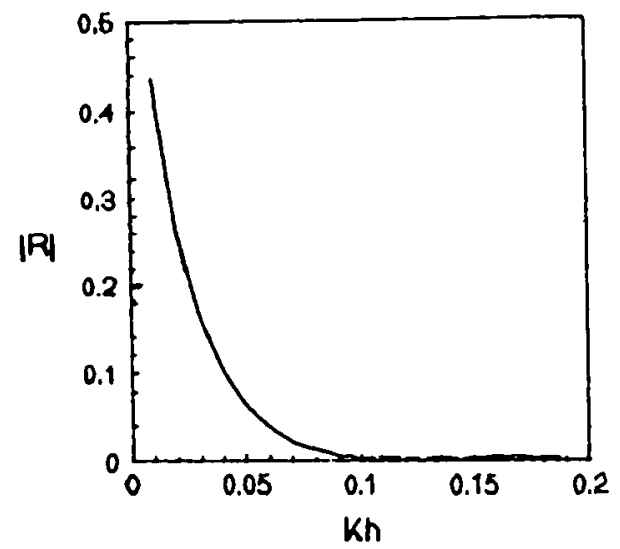

FIGURE 6A. Reflection of water waves by a wall with two gaps $\mu=1.9, \alpha=12.5$

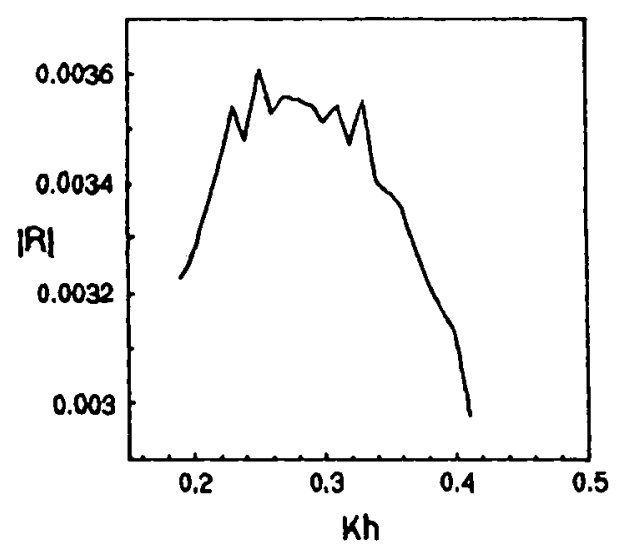

FIGURE 6B. Reflection of water waves by a wall with two gaps $\mu=1.9, \alpha=12.5$

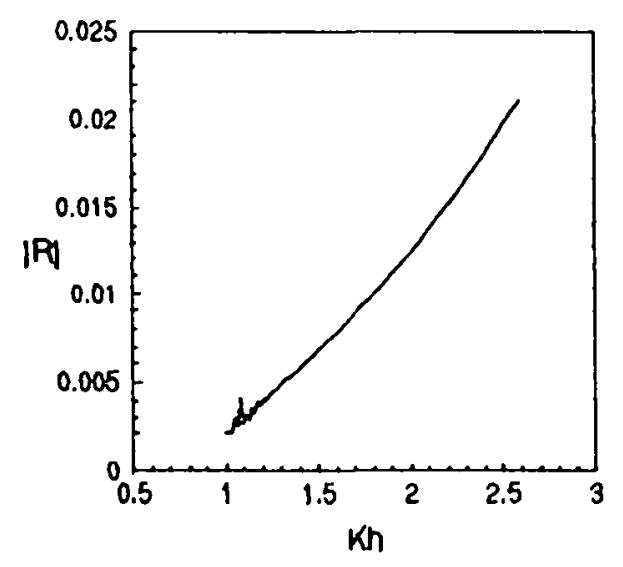

FIGURE 6c. Reflection of water waves by a wall with two gaps $\mu=1.9, \alpha=12.5$

For case (ii), $a_{1}=(2-\mu) h / 2, b_{1}=(2+\mu) h / 2, a_{2}=2 h, b_{2}=2 \alpha h$. For this case $|R|$ is plotted in Figures $6 \mathrm{a}, 6 \mathrm{~b}, 6 \mathrm{c}$ and $7 \mathrm{a}, 7 \mathrm{~b}$ against $K h$ for various values of the parameter.

For $\mu=1.9, \alpha=12.5$, it is observed from Figure 6 a that $|R|$ has a moderate value (not too large) for small values of $K h$, which decreases rapidly and becomes close to zero value whence it exhibits some sort of oscillatory behaviour (see Figures $6 b, 6 c$ ) and finally increases slowly as the wave number $K h$ increases (see Figure $6 \mathrm{c}$ ). 


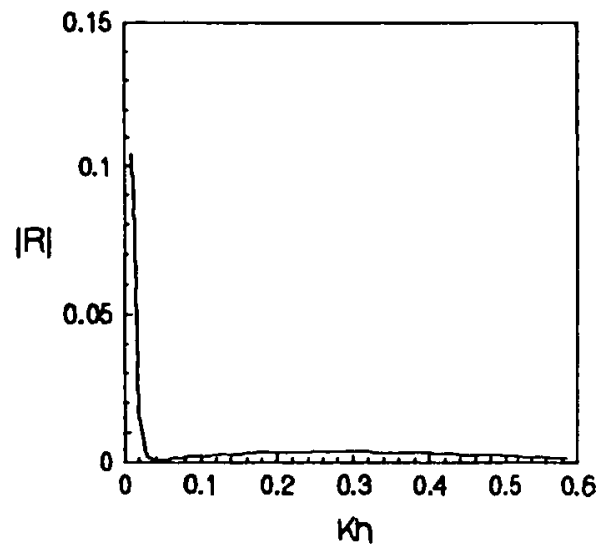

FIGURE 7A. Reflection of water waves by a wall with two gaps $\mu=1.9, \alpha=50$

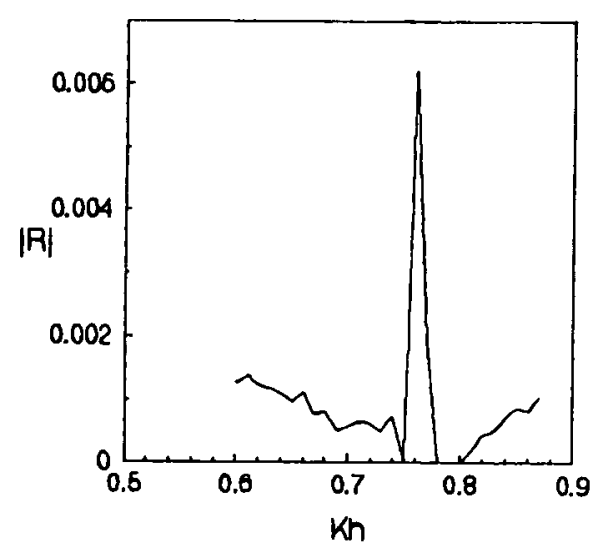

FIGURE 7B. Reflection of water waves by a wall with two gaps $\mu=1.9, \alpha=50$

However, for $\alpha=50$, (see Figures 7a, 7b) it is observed that $|R|$ is very small and exhibits small oscillations for certain ranges of $K h$. It is interesting to observe in this case that there are certain values of $K h$ for which $|R|=0$ (see Figure 7b). This shows that, for large gaps, when the ratio of the lengths of the second gap to the first gap is large, the reflection coefficient is very small for small values of the wave number and there exist certain wave numbers for which the incident wave is totally transmitted through the gaps in the wall.

The behaviour of the reflection coefficient for various values of the wave number has been depicted in Figures 4,5,5a,6a,b,c and 7a,b for cases (i) and (ii) mentioned above for a two-gap problem. For a many-gap problem, a similar analysis can be carried out, and it is possible that a qualitatively similar phenomenon can also be observed in that case, although that has not been analysed here.

\section{Appendix}

Here we solve the integral equation

$$
\int_{B} \frac{2 t h(t)}{t^{2}-q^{2}} d t=c \text { for } q \in B
$$

where $B=\cup_{j=1}^{n+1} B_{j}$ and $c=c_{i}$ for $q \in B_{i}, i=1,2, \ldots, n+1$ and

$$
h(t)=\left\{\begin{array}{lll}
\mathrm{O}\left(\left|t^{2}-a_{i}^{2}\right|^{-1 / 2}\right) & \text { as } & t \rightarrow a_{i} \\
\mathrm{O}\left(\left|t^{2}-b_{i}^{2}\right|^{-1 / 2}\right) & \text { as } & t \rightarrow b_{i}, i=1,2, \ldots, n .
\end{array}\right.
$$


We substitute

$$
t^{2}=u, \quad q^{2}=v
$$

so that (6.1) becomes

$$
\int_{D} \frac{h_{1}(u)}{u-v} d u=c, \quad v \in D
$$

where $D=\cup_{j=1}^{n+1} D_{j}, D_{1}=\left(0, d_{1}\right), D_{j}=\left(e_{j-1}, d_{j}\right), j=1,2, \ldots, n, D_{n+1}=\left(e_{n}, \infty\right)$, $d_{j}=a_{j}^{2}, e_{j}=b_{j}^{2}$,

$$
h_{1}(u)=h\left(u^{1 / 2}\right)
$$

and

$$
h_{1}(u)=\left\{\begin{array}{lll}
\mathrm{O}\left(\left|u-d_{i}\right|^{-1 / 2}\right) & \text { as } & u \rightarrow d_{i} \\
\mathrm{O}\left(\left|u-e_{i}\right|^{-1 / 2}\right) & \text { as } & u \rightarrow e_{i}, i=1,2, \ldots, n
\end{array}\right.
$$

Let

$$
\omega(z)=\frac{1}{2 \pi i} \int_{D} \frac{h_{1}(u)}{u-z} d u, \quad z=x+i y .
$$

Then $\omega(z)$ is analytic in the complex $z$-plane cut along $D$ so that for $i=1,2, \ldots, n$

$$
\omega(z)=\left\{\begin{array}{lll}
\mathrm{O}\left(\left|z-d_{i}\right|^{-1 / 2}\right) & \text { as } & z \rightarrow d_{i} \\
\mathrm{O}\left(\left|z-e_{i}\right|^{-1 / 2}\right) & \text { as } & z \rightarrow e_{i}
\end{array}\right.
$$

and $|\omega(z)|$ is bounded as $z$ tends to 0 and $\infty$.

By the Plemelj-Sokhotskii formula (see [1])

$$
\omega^{ \pm}(x)= \pm \frac{1}{2} h_{1}(x)+\frac{1}{2 \pi i} \int_{D} \frac{h_{1}(u)}{u-x} d u, \quad x \in D
$$

so that for $x \in D$,

$$
\omega^{+}(x)+\omega^{-}(x)=c /(\pi i)
$$

and

$$
\omega^{+}(x)-\omega^{-}(x)=h_{1}(x)
$$

where

$$
\omega^{ \pm}(x)=\lim _{y \rightarrow 0_{ \pm}} \omega(z)
$$

in equation (6.8) is a Riemann Hilbert problem whose solution (after noting (6.7)) is given by [1] as

$$
\omega(z)=\omega_{0}(z)\left[P_{n-1}(z)+\frac{1}{2 \pi i} \int_{D} \frac{c}{\pi i \omega_{0}^{+}(x)} \frac{d u}{u-z}\right],
$$


where $P_{n-1}(z)$ is a polynomial of degree $n-1$,

$$
\omega_{0}(z)=\frac{z^{1 / 2}}{\prod_{j=1}^{n}\left\{\left(z-d_{j}\right)\left(z-e_{j}\right)\right\}^{1 / 2}}
$$

and

$$
\omega_{0}^{ \pm}(x)=\lim _{y \rightarrow 0_{ \pm}} \omega_{0}(z) .
$$

Again by the Plemelj-Sokhotskii formula,

$$
\omega^{ \pm}(x)=\omega_{0}^{ \pm}(x)\left[P_{n-1}(x) \pm \frac{1}{2 \pi i} \frac{c}{\pi i \omega_{0}^{+}(x)}+\frac{1}{2 \pi i} \int_{D} \frac{c}{\pi i \omega_{0}^{+}(u)} \frac{d u}{u-x}\right]
$$

so that from (6.9) we obtain for $x \in D_{j}, j=1,2, \ldots, n+1$ that

$$
h_{1}(x)=\omega_{0}^{+}(x)\left[2 P_{n-1}(x)-\frac{1}{\pi^{2}} \int_{D} \frac{c}{\omega_{0}^{+}(u)} \frac{d u}{u-x}\right] .
$$

Let us choose $2 P_{n-1}(x)=\pi \sum_{k=1}^{n} p_{k-1} x^{k-1}$, where $p_{0}, p_{1}, \ldots, p_{n-1}$ are $n$ arbitrary constants. Finally, using (6.4), the solution of (6.1) is obtained as

$$
\begin{gathered}
h(v)=\frac{(-1)^{n+1-r}}{R_{r}(v)} \sum_{k=1}^{n}\left[\pi p_{k-1} v^{2 k-2}-\frac{2}{\pi^{2}} c_{k} F_{k}(v)\right], \quad v \in B_{r}, \\
r=1,2, \ldots, n+1
\end{gathered}
$$

where

$$
F_{k}(v)=(-1)^{n+1-k} \int_{B_{k}} \frac{R_{k}(v)}{u-v} d v
$$

and

$$
R_{k}(u)=\prod_{j=1}^{n}\left|\left(u^{2}-a_{j}^{2}\right)\left(u^{2}-b_{j}^{2}\right)\right|^{1 / 2}
$$

\section{Acknowledgement}

The author thanks the referees for their comments and suggestions to improve the paper. She also thanks the National Board for Higher Mathematics for financial support when an earlier version of the paper was prepared, and Dr. B. N. Mandal of the Indian Statistical Institute, Calcutta, for many useful discussions. The paper was revised when the author was visiting the Indian Institute of Science, Bangalore, during June 1994. 


\section{References}

[1] Sudeshna Banerjea and B. N. Mandal, "Solution of a singular integral equation in a double interval arising in the theory of water waves", Appl. Math. Lett. 6 (3) (1993) 81-84.

[2] W. R. Dean, "On the reflexion of surface waves by a submerged vertical plate", Proc. Camb. Phil. Soc. 41 (1945) 231-238.

[3] D. V. Evans, "Diffraction of water waves by a submerged vertical plate", J. Fluid Mech. $\mathbf{4 0}$ (1970) 433-451.

[4] T. H. Havelock, "Forced surface-waves on water", Philos. Mag. 8 (1929) 569-576.

[5] M. Lewin, "The effect of vertical barriers on progressing waves", J. Math. Phys. 42 (1963) 287-300.

[6] C. Macaskill, "Reflexion of water waves by a permeable barrier", J. Fluid Mech. 95 (1979) 141-157.

[7] C. C. Mei, "Radiation and scattering of transient gravity waves by vertical plates", Quart.J.Mech. Appl. Math. 19 (1966) 417-440.

[8] N. I. Muskhelishvili, Singular integral equations (Noordhoff, Groningen, 1963).

[9] D. Porter, "The transmission of surface waves through a gap in a vertical barrier", Proc. Camb. Phil. Soc. 71 (1972) 411-421.

[10] D. Porter, "The radiation and scattering of surface waves by vertical barriers", J. Fluid Mech. 63 (1974) 625-634.

[11] E. O. Tuck, "Transmission of water waves through small apertures", J. Fluid Mech. 49 (1971) 65-74.

[12] F. Ursell, "The effect of a fixed vertical barrier on surface waves in deep water", Proc. Camb. Phil. Soc. 43 (1947) 374-382. 